\title{
Relación entre la calidad de vida relacionada con la salud y la ansiedad/ depresión en pacientes en hemodiálisis crónica
}

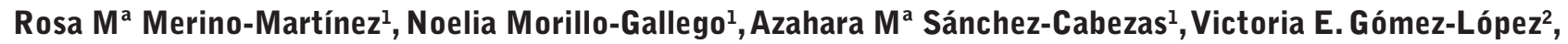 \\ Rodolfo Crespo-Montero ${ }^{1,2,3}$ \\ ${ }^{1}$ Departamento de Enfermería. Facultad de Medicina y Enfermería. Universidad de Córdoba. España \\ 2 Servicio de Nefrología. Hospital Universitario Reina Sofía de Córdoba. España \\ ${ }^{3}$ Instituto Maimónides de Investigación Biomédica de Córdoba. España
}

Como citar este artículo:

Merino-Martínez RM, Morillo-Gallego N, Sánchez-Cabezas AM, Gómez-López VE, Crespo-Montero R. Relación entre la calidad de vida relacionada con la salud y la ansiedad/depresión en pacientes en hemodiálisis crónica. Enferm Nefrol. 2019

Jul-Sep;22(3):274-83

\section{Resumen}

Objetivo: El objetivo del presente estudio fue analizar la Calidad de Vida Relacionada con la Salud en pacientes hemodiálisis y su relación con la ansiedad y la depresión.

Pacientes y Método: Se estudiaron 122 pacientes en hemodiálisis, con una edad de $66,47 \pm 14,2$ años (34,4\% mujeres). Se realizó un estudio descriptivo y transversal, en el área de influencia del Servicio de Nefrología del Hospital Reina Sofía de Córdoba. Se estudió la Calidad de Vida Relacionada con la Salud (cuestionario KDQ0L-SF), Ansiedad y Depresión (cuestionario Escala de Ansiedad y Depresión de Goldberg) y la comorbilidad asociada.

Resultados: En el KDQOL, las dimensiones más afectadas fueron: Situación Laboral, Carga de la Enfermedad Renal, Función Sexual y Sueño. En las dimensiones del cuestionario SF, las áreas más afectadas fueron: Rol Físico, Salud General y Función Física. El 42,6\% de los pacientes presentaron probabilidad de tener ansiedad, el $52,5 \%$ depresión, y el $32,8 \%$ presentaban la probabilidad de padecer ambas alteraciones (ansiedad y depresión). La ansiedad y la depresión se asociaron de forma significativa a peores puntuaciones en todas las dimensiones del cuestionario KDQOL-SF.

Correspondencia:

Rosa Maa Merino Martínez

C/ Blanca, 9. 14550 Montilla. Córdoba

E-mail: rosamerinomartinez@gmail.com
Conclusiones: Los pacientes en hemodiálisis presentan peor calidad de vida que la población sana de referencia, especialmente en las dimensiones Carga de la enfermedad renal, Sueño, Salud general, Vitalidad, Función física y Rol físico. Esta disminución de la calidad de vida está relacionada con la probabilidad de padecer ansiedad y/o depresión. El sexo femenino, la comorbilidad asociada y el tiempo en hemodiálisis, se asocian también a peor calidad de vida.

PALABRAS CLAVE: calidad de vida relacionada con la salud; hemodiálisis; ansiedad; depresión.

\section{Relationship between health-related quality of life and anxiety / depression in patients on chronic hemodialysis}

\section{Abstract}

Objective: The aim of the present study was to analyze the health-related quality of life in hemodialysis patients and their relationship with anxiety and depression.

Material and Method: One hundred and twenty-two hemodialysis patients were studied, with an age of $66.47 \pm 14.2$ years ( $34.4 \%$ women). A descriptive and cross-sectional study was carried out in around the Nephrology Unit of the Reina Sofía University Hospital of Cordoba. The health-related quality of life (KDQOLSF questionnaire), anxiety and depression (Goldberg 
Anxiety and Depression Scale questionnaire) and associated comorbidity were studied.

Results: In the KDQOL scale, the most affected dimensions were: Work Status, Kidney Disease Burden, Sexual Function and Sleep. In the dimensions of the SF questionnaire, the most affected areas were: Physical Role, General Health and Physical Function. $42.6 \%$ of the patients presented probability of having anxiety, $52.5 \%$ depression, and $32.8 \%$ had the probability of suffering both alterations (anxiety and depression). Anxiety and depression were significantly associated with worse scores in all dimensions of the KDQOL-SF questionnaire.

Conclusions: Hemodialysis patients have a worse quality of life than the healthy reference population, especially in the Kidney Disease Burden, Sleep, General health, Vitality, Physical Function and Physical Role dimensions. This decrease in the quality of life is related to the probability of suffering anxiety and/or depression. Female sex, associated comorbidity and length of time on hemodialysis are also associated with a worse quality of life.

KEYWORDS: health-related quality of life; hemodialysis; anxiety; depression.

\section{Introducción}

La enfermedad renal crónica terminal (ERCA) es una alteración crónica cuya incidencia va aumentando progresivamente en nuestro país, lo que supone un problema sanitario, social y económico de especial relevancia; y que en su último estadio precisa un tratamiento renal sustitutivo (TRS) ${ }^{1}$. En nuestro país, la mayoría de los pacientes inician TRS con hemodiálisis (HD). Según el Registro de Diálisis y Trasplantes de 2017, la prevalencia se situó en 1284,2 pacientes por millón de población, mientras que la incidencia de la ERCA es de 141,0 pacientes por millón de población, siendo más del $50 \%$ los pacientes mayores de 65 años $^{2}$. Además de la edad, estos pacientes tienen diferentes patologías asociadas (diabetes, insuficiencia cardiaca, enfermedades vasculares, etc.), que condiciona un grado mayor de dependencia y una alta morbimortalidad con frecuentes ingresos hospitalarios ${ }^{3,4}$. Como consecuencia de todo esto, la calidad de vida de estos pacientes, se va a afectada de forma importante ${ }^{5,6}$.

Tradicionalmente, el concepto de salud se ha relacionado con padecer alguna enfermedad, en el que el abordaje se centraba en la curación de la misma y en el alivio del dolor; sin embargo, con el paso del tiempo y los propios avances de este campo, esta definición de salud ha quedado incompleta, definiéndose en la actualidad la salud como el completo estado de bienestar físico, espiritual, emocional, mental y social. Esta visión ampliada de la salud ha hecho que se incorporen conceptos como el de calidad de vida relacionada con la salud $(\text { CVRS })^{7}$. La CVRS, es un concepto multidimensional que ha sido definido como la evaluación subjetiva que realiza un individuo sobre el impacto de la enfermedad y su tratamiento sobre la dimensión física, psicológica y social, valorando el impacto sobre la funcionalidad y el bienestarb.

El empleo de medidores de CVRS es especialmente relevante en patologías con tendencia a la cronicidad, como es el caso de la ERCA en tratamiento de diálisis. Aunque los avances, tanto técnicos como sanitarios, han puesto de manifiesto una amplia mejoría en la calidad de vida de estos enfermos, diversos estudios describen una disminución destacable de la calidad de vida en comparación con la población general ${ }^{8}$. Estas diferencias son especialmente pronunciadas en un amplio abanico de cambios físicos, psicológicos y sociales; de este modo, las variables psicosociales tienen un papel fundamental en la atención integral del paciente en HD. Además, la ERCA, como otras enfermedades crónicas, pueden tratarse, pero no tiene curación; y por lo tanto, el tratamiento y los cuidados hacia este perfil de pacientes, no solo deben observar resultados objetivos, sino y al mismo nivel, las percepciones de los mismos sobre su calidad de vida?.

Por otro lado, diferentes autores coinciden, que tanto la ansiedad como la depresión son variables fundamentales y determinativos en la CVRS ${ }^{10,11}$ en HD. Los resultados publicados por Perales-Montilla et al, sitúan a la depresión como un predictor fundamental de CVRS, afectando a las dimensiones Función Física, Rol Físico, Vitalidad, Función Social y Salud Mental; además de evidenciar una relación inversamente proporcional en lo que respecta a la CVRS y la ansiedad ${ }^{12}$. Los cambios tanto a nivel psicológico como en CVRS que afectan a estos pacientes, no solo están relacionados con el propio tratamiento de diálisis, sino también con otras variables intrínsecas como puede ser la edad, género 0 ingresos hospitalarios ${ }^{13}$.

Los pacientes en tratamiento de HD, se caracterizan por sufrir en un plazo breve de tiempo un conjunto de cambios bruscos en su vida ligados a la enfermedad y al propio tratamiento que pueden debutar en trastornos ansiosos-depresivos. El estado emocional del paciente (ansiedad, depresión, estrés...) influye notoriamente en el transcurso de la enfermedad, relacionándose con un incremento de la mortalidad y de la morbilidad ${ }^{14}$. 
Por lo tanto, dada la importancia y la asociación entre ansiedad y/o depresión y CVRS en pacientes en tratamiento en HD, sería interesante abordar la relación existente entre las variables descritas. Conocer la CVRS en estos pacientes, es fundamental desde el punto de vista de la atención integral que se les presta.

Por todo ello, el objetivo del presente estudio ha sido analizar la CVRS de los pacientes con ERCA en HD periódicas y su relación con la ansiedad/depresión, con los siguientes objetivos específicos:

1. Determinar la relación entre la ansiedad/depresión y la CVRS de los pacientes en HD.

2. Identificar las variables más influyentes en la calidad de vida de estos pacientes.

\section{Pacientes y Método}

\section{Ámbito de estudio}

El estudio ha sido desarrollado en el área de influencia del Servicio de Nefrología del Hospital Universitario Reina Sofía (HURS) de Córdoba. Se incluyeron en el estudio todos los pacientes tratados en la Unidad de Diálisis Hospitalaria (UDH), el Centro Periférico de Diálisis Público "Perpetuo Socorro" (CPDP) y el Centro Concertado de Diálisis "San Rafael" de Fresenius Medical Care (CCD). Se llevó a cabo en los meses de enero a abril de 2019.

\section{Diseño}

Estudio observacional, descriptivo de corte transversal.

\section{Población y muestra}

La población de estudio fueron los pacientes con ERCA en tratamiento con HD periódicas en la UDH, CPDP y CCD.

Para ello, se incluyó a todos los pacientes incluidos en el programa de HD de estas unidades, que de acuerdo a los criterios de inclusión, aceptaron participar en el estudio, tras informarles del objetivo y previo consentimiento informado. Se obtuvo una muestra final de 122 pacientes.

\section{Criterios de inclusión}

Los criterios de inclusión en este estudio fueron: pacientes mayores de 18 años, que reunieran las condiciones óptimas tanto físicas como mentales para responder a las preguntas de los cuestionarios.

\section{Variables del estudio}

Variable de resultado: CVRS y ansiedad/depresión.

Otras variables analizadas fueron: edad, sexo, tiempo en diálisis y comorbilidad.

\section{Instrumentos de medida}

Como instrumento de medida de la CVRS se empleó el cuestionario validado Kidney Disease and Quality of Life (KDQOL-SF), siendo éste una adaptación del cuestionario SF-36 para pacientes con ERCA. Este cuestionario autoadministrado está provisto de 78 ítems en relación a 19 dimensiones, vinculadas 11 de ellas a escalas específicas para enfermedades renales, como son; Síntomas/ problemas, Efectos de la enfermedad renal, Carga de la enfermedad renal, Situación laboral, Función cognitiva, Calidad de las relaciones sociales, Función sexual, Sueño, Apoyo social, Actitud del personal de diálisis y Satisfacción del paciente, y 8 ítems del SF-36, correspondientes a: la Función física, Rol físico, Dolor, Salud general, Bienestar emocional, Rol emocional, Función social y Vitalidad. Una vez corregido la puntuación bruta para cada dimensión fluctúa entre 0 (peor CVRS) a 100 (mejor CVRS).

Se utilizó el Índice de Comorbilidad de Charlson (ICC) para realizar el análisis de la comorbilidad.

Para el cribado de la ansiedad y la depresión se utilizó la "Escala de Ansiedad y Depresión de Goldberg". Se trata de un cuestionario heteroadministrado con dos subescalas, una de ansiedad y otra de depresión. Cada una de las subescalas se estructura en 4 ítems iniciales de despistaje y un segundo grupo de preguntas compuesto de 5 ítems que se formulan si se obtienen respuestas positivas a las preguntas de despistaje (2 o más en la Subescala de Ansiedad, 10 más en la Subescala de Depresión. Los puntos de cortes son $\geq 4$ en el caso de la escala de ansiedad, y $\geq 2$ para la depresión. Para la población geriátrica ( $>75$ años) se utilizó el punto corte de $\geq 6$, propuesto por los autores del cuestionario. 


\section{Recogida de datos}

Los datos fueron recogidos entre los meses de enero y marzo, durante el desarrollo de las sesiones de HD, para no añadir tiempo extra al paciente por el hecho de participar en el estudio. Aunque el KDQOL-SF es un cuestionario autoadministrado, por una estrategia de optimización de los tiempos, y hacerlo conjuntamente con la Escala de Ansiedad y Depresión de Goldberg, la primera autora del estudio realizó todas las entrevistas de ambos cuestionarios a todos los pacientes; y sobre todo, para evitar sesgos de interpretación en las respuestas.

En todo momento, se garantizó la confidencialidad de los datos recogidos.

\section{Aspectos éticos}

La información recogida ha sido tratada con arreglo a la actual Ley Orgánica 15/1999 de 13 diciembre, sobre "Protección de datos de carácter personal", y con el único fin del desarrollo de esta investigación.

La participación en el estudio ha sido en todo momento de carácter voluntaria y anónima, respetando en cualquier punto de su desarrollo que cualquier usuario decidiese abandonar la entrevista, sin necesidad de justificar el motivo por el cuál dejaba de participar, ni ser motivado a finalizar dichos cuestionarios.

El desarrollo del presente estudio se puso en marcha una vez recibido el informe favorable del Comité de Ética e Investigación del HURS, con código: CVRSADPHC2018.

\section{Análisis estadístico}

Para las variables cualitativas se utilizó una distribución de frecuencias, mientras que para las variables numéricas se usó la media \pm la desviación estándar de la media y en algunos casos la mediana. Para la comparación entre variables se utilizó la t de Student para medias independientes o pruebas no paramétricas según siguieran o no una distribución normal. También se realizaron correlaciones con el test de Pearson. Se aceptó significación estadística para $p<0,05$. El análisis estadístico se ha realizado en un paquete estadístico SSPS 25.0 para Windows.

\section{Resultados}

Se estudiaron un total de 122 pacientes, con una edad media de $66,47 \pm 14,2$ años; distribuyéndose en 80 hombres
$(65,6 \%)$ y 42 mujeres (34,4\%). La mediana del tiempo en $\mathrm{HD}$ fue de 39,5 meses, con un rango intercuartil de 71,25 meses. El $74,6 \%$ de los pacientes se dializaban a través de una fístula arteriovenosa (FAV) y el $25,4 \%$, lo hacían a través de un catéter venoso central para HD tunelizado (CVT). Respecto a la distribución por centros de diálisis, 27 pacientes $(22,1 \%)$ pertenecían a la UDH, 50 pacientes $(41 \%)$ al CPDP y 45 pacientes $(36,9 \%)$ al CCD. En la Tabla 1 se representan los resultados del cuestionario KDQOL-SF/SF-36.

Tabla 1. Valores de las dimensiones del KDQOL y del SF-36.

\begin{tabular}{|l|c|c|}
\hline $\begin{array}{l}\text { Escalas Específicas para } \\
\text { Enfermedades Renales }\end{array}$ & Media y DE & Mín-Máx \\
\hline Listado de síntomas/problemas & $72,37 \pm 18,06$ & $0-100$ \\
\hline Efectos de la enfermedad renal & $59,62 \pm 22,93$ & $0-100$ \\
\hline Carga de la enfermedad renal & $40,11 \pm 27,16$ & $0-100$ \\
\hline Situación laboral & $27,25 \pm 27,84$ & $0-100$ \\
\hline Función cognitiva & $80,90 \pm 19,36$ & $20-100$ \\
\hline Calidad de las relaciones sociales & $83,87 \pm 20,3$ & $20-100$ \\
\hline Función sexual & $50,37 \pm 37$ & $0-100$ \\
\hline Sueño & $58,92 \pm 27,06$ & $0-100$ \\
\hline Apoyo social & $80,50 \pm 26,24$ & $0-100$ \\
\hline Actitud del personal de diálisis & $88,6270 \pm 21,44$ & $0-100$ \\
\hline Satisfacción del paciente & $87,6909 \pm 18,28$ & $33,33-100$ \\
\hline Ítems del SF-36 & Media y DT & Mín-Máx \\
\hline Función física & $47,66 \pm 31,93$ & $0-100$ \\
\hline Rol físico & $29,92 \pm 39,84$ & $0-100$ \\
\hline Dolor & $61,11 \pm 35,95$ & $0-100$ \\
\hline Salud general & $40,99 \pm 20,27$ & $0-95$ \\
\hline Bienestar emocional & $64,30 \pm 25,59$ & $8-100$ \\
\hline Rol emocional & $55,60 \pm 47,41$ & $0-100$ \\
\hline Función social & $66,63 \pm 34,13$ & $12,50-100$ \\
\hline Vitalidad & $48,93 \pm 27,33$ & $0-100$ \\
\hline
\end{tabular}

DE: Desviación Estándar de la media

Al comparar hombres y mujeres, no se encontraron diferencias estadísticamente significativas, en la Escala Específica para Enfermedades Renales. En la dimensiones del cuestionario SF-36, se encontraron diferencias significativas para tres de las ocho dimensiones, correspondientes a Función física, Dolor y Vitalidad, tal como muestra la Tabla 2.

Respecto al acceso vascular, se encontraron diferencias significativas en las dimensiones Listado de síntomas/ problemas $(74,8 \pm 15,8$-FAV- frente a $65 \pm 22$-CVT-, $p<0,05)$ y Efectos de la enfermedad renal $(62,5 \pm 21,9$ -FAV- frente a $51,1 \pm 23,8$-CVT-, $p<0,05)$. 
Tabla 2. Valores de las dimensiones del KDQOL y del SF-36.

\begin{tabular}{|l|c|c|c|}
\hline $\begin{array}{l}\text { Escalas Específicas } \\
\text { para Enfermedades Renales }\end{array}$ & Hombres & Mujeres & Valor de p \\
\hline Listado de síntomas/problemas & $74,03 \pm 17,58$ & $69,2 \pm 18,74$ & NS \\
\hline Efectos de la enfermedad renal & $57,73 \pm 21,65$ & $63,2 \pm 25,07$ & NS \\
\hline Carga de la enfermedad renal & $39,69 \pm 25,36$ & $40,92 \pm 30,62$ & NS \\
\hline Situación laboral & $27,5 \pm 28,3$ & $26,79 \pm 27,27$ & NS \\
\hline Función cognitiva & $80,29 \pm 20,5$ & $82,1 \pm 17,14$ & NS \\
\hline Calidad de las relaciones sociales & $82,44 \pm 20,1$ & $86,59 \pm 20,65$ & NS \\
\hline Función sexual & $50,78 \pm 34,75$ & $49,4 \pm 41,5$ & NS \\
\hline Sueño & $59,64 \pm 26,75$ & $57,56 \pm 27,92$ & NS \\
\hline Apoyo social & $81,25 \pm 25,63$ & $79,1 \pm 27,65$ & NS \\
\hline Actitud del personal de diálisis & $87,81 \pm 20,21$ & $90,18 \pm 24$ & NS \\
\hline Satisfacción & $87,21 \pm 18,78$ & $88,61 \pm 17,48$ & NS \\
\hline Ítems del SF-36 & Hombres & Mujeres & Valor de p \\
\hline Función Física & $53,81 \pm 31,21$ & $35,95 \pm 30,29$ & p<0,01) \\
\hline Rol Físico & $33,44 \pm 41,71$ & $23,21 \pm 35,52$ & NS \\
\hline Dolor & $68,72 \pm 34,11$ & $46,61 \pm 35,26$ & $\mathrm{p}<0,001$ \\
\hline Salud general & $42,83 \pm 20,37$ & $37,5 \pm 19,86$ & NS \\
\hline Bienestar emocional & $67,5 \pm 25,20$ & $58,19 \pm 25,52$ & NS \\
\hline Rol emocional & $61,25 \pm 47,27$ & $44,84 \pm 46,34$ & NS \\
\hline Función social & $70,56 \pm 33,16$ & $59,17 \pm 35,1$ & NS \\
\hline Vitalidad & $54,5 \pm 28,21$ & $38,33 \pm 22,24$ & $\mathrm{p}<0,01$ \\
\hline
\end{tabular}

de hombres) y 16 mujeres $(38,1 \%$ del total de mujeres).

En relación al sexo, los casos de probable Ansiedad, se dieron en 21 mujeres $(50 \%$ del total de mujeres) y 31 hombres $(38,75 \%$ del total de hombres). Mientras, en casos de probable Depresión, fueron 39 hombres $(48,75 \%$ del total de hombres) y 25 mujeres $(59,52 \%$ del total de mujeres entrevistadas).

Para la Subescala de Ansiedad, se hallaron correlaciones estadísticamente significativas con todas las dimensiones para las escalas específicas para enfermedades renales del cuestionario KDQOL-SF, exceptuando; Actitud y Satisfacción. Del mismo modo, para la Subescala Depresión, se encontraron correlaciones estadísticamente significativas con todas las dimensiones para las escalas específicas para enfermedades renales del cuestionario KDQOL-SF, a excepción; situación laboral, actitud y satisfacción (Tabla 3).
Para comparar los resultados del cuestionario SF36, respecto a la población general, se utilizaron los valores poblacionales para el mismo grupo de edad de este cuestionario (Figura 1).

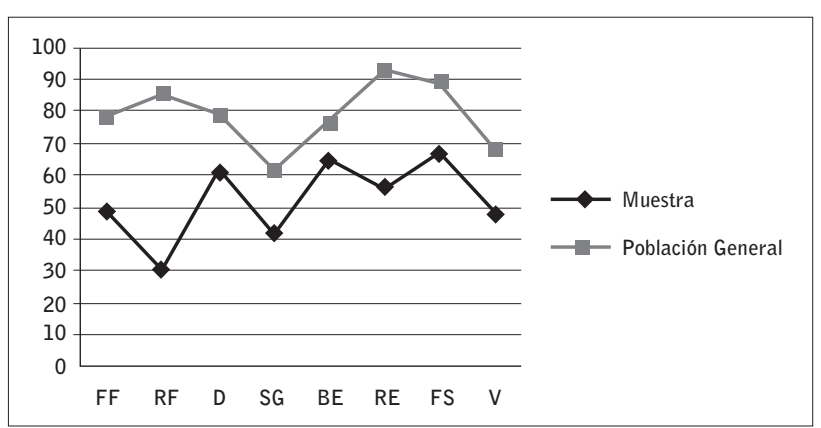

FF: Función Física, RF: Rol Físico, D:Dolor, SG: Salud General, BE: Bienestar Emocional, RE: Rol Emocional, FS: Función Social, V: Vitalidad.

Figura 1. Valores medios de las dimensiones de la población estudiada y la población general española.

En cuanto a la prevalencia de probables casos de ansiedad y depresión, 52 pacientes $(42,6 \%)$ y 64 pacientes $(52,5 \%)$ presentaron una alta probabilidad de ansiedad y depresión respectivamente. Con ambas afectaciones (ansiedad y depresión) se encontraron 40 pacientes $(32,79 \%)$, 24 hombres $(30 \%$ del total
Tabla 3. Correlación entre Subescala de Ansiedad y Subescala de Depresión para las dimensiones del cuestionario KDQ0L-SF y el en el SF-36.

\begin{tabular}{|c|c|c|}
\hline $\begin{array}{l}\text { Escalas Específicas } \\
\text { para Enfermedades Renales }\end{array}$ & $\begin{array}{l}\text { Subescala } \\
\text { Ansiedad }\end{array}$ & $\begin{array}{l}\text { Subescala } \\
\text { Depresión }\end{array}$ \\
\hline Listado de síntomas/problemas & $r=-0,465 ; p<0,01$ & $r=-0,552 ; p<0,01$ \\
\hline Efectos de la enfermedad renal & $r=-0,387 ; p<0,01$ & $r=-0,416 ; p<0,01$ \\
\hline Carga de la enfermedad renal & $r=-0,445 ; p<0,01$ & $r=-0,447 ; p<0,01$ \\
\hline Situación laboral & $r=-0,260 ; p<0,01$ & $r=-0,118 ; N S$ \\
\hline Función cognitiva & $r=-0,503 ; p<0,01$ & $r=-0,439 p<0,01$ \\
\hline Calidad de las relaciones sociales & $r=-0,491 ; p<0,01$ & $r=-0,304 ; p<0,01$ \\
\hline Función sexual & $r=-0,188 ; p<0,01$ & $r=-0,195 ; p<0,05$ \\
\hline Sueño & $r=-0,463 ; p<0,01$ & $r=-0,411 ; p<0,01$ \\
\hline Apoyo social & $r=-0,297 ; p<0,01$ & $r=-0,187 ; p<0,05$ \\
\hline Actitud & $r=-0,118 ; N S$ & $r=-0,051 ; N S$ \\
\hline Satisfacción & $r=-0,111 \mathrm{NS}$ & $r=-0,031 ; N S$ \\
\hline Ítems del SF-36 & Subescala Ansiedad & Subescala Depresión \\
\hline Función física & $r=-0,216 ; p<0,05$ & $r=-0,360 ; p<0,01$ \\
\hline Rol físico & $r=-0,258 ; p<0,01$ & $r=-0,439 ; p<0,01$ \\
\hline Dolor & $r=-0,285 ; p<0,01$ & $r=-0,313 ; p<0,01$ \\
\hline Salud general & $r=-0,198 ; p<0,05$ & $r=-0,300 ; p<0,01$ \\
\hline Bienestar emocional & $r=-0,610 ; p<0,01$ & $r=-0,612 ; p<0,01$ \\
\hline Rol emocional & $r=-0,354 ; p<0,01$ & $r=-0,463 ; p<0,01$ \\
\hline Función social & $r=-0,248 ; p<0,01$ & $r=-0,396 ; p<0,01$ \\
\hline Vitalidad & $r=-0,363 ; p<0,01$ & $r=-0,512 ; p<0,01$ \\
\hline
\end{tabular}


Respecto a los Ítems del SF-36, se encontró correlación estadísticamente significativa con las Subescalas de Ansiedad y Depresión con todas sus dimensiones.

La media del ICC para la muestra fue de $6,11 \pm 2,2$ puntos, siendo por tanto, la comorbilidad del grupo alta (ICC $>3$ ). EI ICC fue similar entre hombres y mujeres (6,1 puntos). No se encontró relación entre el ICC, y las Subescalas de Ansiedad y Depresión.

Se encontró una relación estadísticamente significativa entre el ICC y la variable Función física ( $r=-0,377$; $\mathrm{p}<0,01$ ) de las dimensiones del SF-36; no encontrándose ninguna otra relación estadística.

Entre la edad y las variables del KDQOL-SF, solo se encontraron relaciones significativas con la Función física $(r=-0,369 ; p<0,01)$. Entre las variables Edad e ICC se encontró relación significativa $(r=0,596, p>0,01)$.
El total de población geriátrica ( 275 años) fue 38 personas $(31,15 \%)$. Comparando la puntuación media para ICC, entre los pacientes geriátricos y no geriátricos, la diferencia fue significativa, $(7,47$ frente a 5,50, $p<0,05)$. Al comparar los valores del cuestionario KDQOL-SF entre la población no geriátrica y geriátrica, sólo se encontró diferencias estadísticamente significativas en la Función física $(p<0,001)$.

Analizando el tiempo en HD de los pacientes, se encontraron relaciones estadísticamente significativas con el KDQ0L-SF para Efectos de la enfermedad renal $(r=-0,193$; $p<0,05)$, Situación laboral $(r=-0,186 ; p<0,05)$ y Sueño $(r=-0,249 ; p<0,01)$. Mientras que, para el SF-36 solo encontraron relaciones estadísticamente significativas con la Salud general $(r=-0,238 ; p<0,01)$.

También se compararon las distintas variables entre los 3 centros de HD, tal como puede apreciarse en la Tabla 4.

Tabla 4. Comparación de las distintas variables entre los centros de HD.

\begin{tabular}{|c|c|c|c|c|}
\hline & UDH & CPDP & CCD & Valor de $\mathbf{p}$ \\
\hline Edad & $67,30 \pm 13,1$ & $64,38 \pm 15,7$ & $68,29 \pm 13,03$ & NS \\
\hline ICC & $6,33 \pm 2,22$ & $5,64 \pm 1,99$ & $6,51 \pm 2,36$ & $p<0,05$ \\
\hline Tiempo HD & $61,44 \pm 77,68$ & $62,08 \pm 66,59$ & $58,51 \pm 50,93$ & NS \\
\hline Sub.Ansiedad & $3,96 \pm 3,09$ & $2,8 \pm 3$ & $2,87 \pm 2,72$ & NS \\
\hline Sub.Depresión & $3,93 \pm 2,95$ & $2,9 \pm 2,44$ & $2,69 \pm 2,52$ & NS \\
\hline Escalas Específicas para Enfermedades Renales & UDH & CPDP & CCD & \\
\hline Listado de síntomas/problemas & $63,66 \pm 22,74$ & $74 \pm 15,96$ & $75,78 \pm 15,68$ & NS \\
\hline Efectos de la enfermedad renal & $49,54 \pm 21,51$ & $57 \pm 22,5$ & $68,57 \pm 21,37$ & $p<0,01$ \\
\hline Carga de la enfermedad renal & $29,17 \pm 27,08$ & $37,5 \pm 25,72$ & $49,58 \pm 26,19$ & $p<0,01$ \\
\hline Situación laboral & $18,51 \pm 24,6$ & $25,5 \pm 28,34$ & $34,44 \pm 27,84$ & NS \\
\hline Función cognitiva & $77,65 \pm 19,95$ & $79,6 \pm 21,1$ & $84,3 \pm 16,71$ & NS \\
\hline Calidad de las relaciones sociales & $77,47 \pm 24,57$ & $82,2 \pm 19,65$ & $89,56 \pm 16,87$ & $p<0,05$ \\
\hline Función sexual & $36,57 \pm 41,15$ & $61 \pm 44,87$ & $51,67 \pm 37,84$ & $p<0,05$ \\
\hline Sueño & $51,34 \pm 26,8$ & $58,05 \pm 27,86$ & $64,44 \pm 25,65$ & NS \\
\hline Apoyo social & $71,60 \pm 29,89$ & $82 \pm 24,71$ & $84,17 \pm 24,93$ & NS \\
\hline Actitud del personal de diálisis & $90,74 \pm 16,84$ & $87 \pm 22,86$ & $89,17 \pm 22,55$ & NS \\
\hline Satisfacción del paciente & $83,70 \pm 20,82$ & $87,43 \pm 18,02$ & $90,37 \pm 16,85$ & NS \\
\hline Ítems del SF-36 & UDH & CPDP & CCD & \\
\hline Función física & $34,48 \pm 31,28$ & $53,5 \pm 31,22$ & $47,89 \pm 32$ & NS \\
\hline Rol físico & $20,37 \pm 39,25$ & $31,5 \pm 39,01$ & $33,89 \pm 41$ & NS \\
\hline Dolor & $47,41 \pm 41,16$ & $64,05 \pm 32,89$ & $66,06 \pm 35,3$ & NS \\
\hline Salud general & $34,44 \pm 20,01$ & $40,8 \pm 20,59$ & $45,14 \pm 19,43$ & $p<0,05$ \\
\hline Bienestar emocional & $53,03 \pm 27,52$ & $66,4 \pm 23,33$ & $68,71 \pm 25,40$ & $p<0,05$ \\
\hline Rol emocional & $32,1 \pm 45,74$ & $62,33 \pm 45,52$ & $62,22 \pm 46,96$ & $p<0,05$ \\
\hline Función social & $52,68 \pm 34,98$ & $71,45 \pm 32,56$ & $69,67 \pm 34,87$ & $p<0,05$ \\
\hline Vitalidad & $43,15 \pm 31,87$ & $50 \pm 24,41$ & $51,22 \pm 27,61$ & NS \\
\hline
\end{tabular}

Se realizó una comparación de muestras independientes mediante el Test no paramétrico Kruskal Wallis. Posteriormente se compararon 2 a 2 las variables que fueron significativas con el Test U de Mann-Whitney. 
Respecto al ICC se encontraron diferencias significativas entre el CPDP y el CCD $(p<0,01)$ En cuanto a los Efectos de la enfermedad renal se encontraron diferencias entre la UDH y CCD $(p<0,001)$ y el CPDP y el CCD $(p<0,01)$. Igual ocurrió con la Carga de la enfermedad renal, encontrándose diferencias entre la UDH y CCD $(p<0,01)$ y el CPDP y el CCD $(p<0,05)$. En cuanto a la Calidad de las relaciones sociales, se encontraron diferencias entre la UDH y CCD $(p<0,05)$ y el CPDP y el $\operatorname{CCD}(p<0,05)$. En la Función sexual se encontraron diferencias entre UDH y CPDP $(p<0,05)$.

En cuanto a las dimensiones del SF-36, se encontró diferencias significativas en la Salud general, entre la UDH y CCD $(p<0,05)$; en el Bienestar emocional se encontraron diferencias entre la UDH y CPDP $(p<0,05)$ y la UDH y CCD $(p<0,05)$, en el Rol emocional se encontraron diferencias entre la UDH y CPDP $(p<0,01)$ y la UDH y CCD $(p<0,05)$; y en la Función social entre la UDH y CPDP $(p<0,05)$ y la UDH y CCD $(p<0,05)$.

\section{Discusión}

El principal propósito de este estudio fue analizar la relación entre la CVRS y la ansiedad/depresión en el área de influencia del Servicio de Nefrología del Hospital Universitario Reina Sofía, ya que la depresión es una de las principales causas de consulta médica y el trastorno afectivo más prevalente en la población anciana, y la ansiedad un problema de salud que interfiere en el bienestar social, laboral e intelectual y en la propia percepción de calidad de vida de la persona ${ }^{15}$. Además de este objetivo, se pretendió conocer las áreas más afectadas en la CVRS del paciente en HD y la prevalencia de posibles trastornos psicológicos.

En el análisis para las Escalas Específicas para Enfermedades Renales (KDQOL), se observa como dimensiones más afectadas: Situación laboral, Carga de la enfermedad renal, Función sexual y Sueño. Por el contrario, aquellas con mejor resultado fueron: Actitud del personal, Satisfacción del paciente, la Calidad de las relaciones sociales y la Función cognitiva; siendo estos resultados similares a los comunicados en otros estudios ${ }^{16,17,18}$. Las puntuaciones más altas coinciden con la evaluación al personal sanitario, en este caso se pidió que contestaran a las preguntas valorando el grado de amabilidad, interés, apoyo, entre otras cuestiones, por lo tanto, refleja un alto grado de satisfacción que sigue una línea similar a la literatura encontrada ${ }^{19,20}$. Como hipótesis a la diferencia de puntuación entre la Carga de la enfermedad renal en comparación con el Listado de síntomas y Efectos de la enfermedad renal, podría justificarse porque la primera evalúa las percepciones subjetivas en torno a la enfermedad renal, sin tener en cuenta los síntomas y los efectos en las vida cotidiana.

Aunque la Situación laboral es la dimensión más afectada, este dato no es representativo, ya que la inmensa mayoría de los entrevistados fueron jubilados, por lo que el resultado se encuentra condicionando por la situación laboral y la propia edad avanzada.

La Función sexual, que en ambos sexos refiere una de las puntuaciones más bajas, se encuentra relacionada de una manera significativa con la ansiedad y la depresión. EI estudio de Lew-Starowicz et al. sitúa la ansiedad en los hombres y la depresión en las mujeres como predictores de una la baja calidad de vida sexual. Por lo tanto, en consonancia con nuestros resultados, se pone de manifiesto una asociación estadística entre la función sexual, la ansiedad y depresión y la CVRS ${ }^{21}$.

Por otra parte, para el cuestionario SF-36, las áreas más afectadas fueron: Rol físico, Salud general y Función física. Mientras que las dimensiones que mejor puntuaron: Función social, Bienestar social y Dolor, en la línea de lo publicados por otros autores 22,23 .

Cuando se comparó hombres y mujeres, no se encontraron diferencias estadísticamente significativas, en la Escala Específica para Enfermedades Renales. Sin embargo, en la dimensiones del cuestionario SF-36, presentaron peor calidad de vida las mujeres, siendo las diferencias significativas en las dimensiones Función física, Dolor y Vitalidad; habiéndose destacado ya en diferentes estudios la

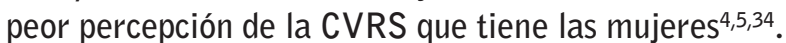

Respecto al acceso vascular, aunque en algunos estudios se ha destacado mejores resultados en la calidad de vida en los pacientes que se dializan con FAV respecto a los pacientes que lo hacen a través de un $\mathrm{CVT}^{24,25}$, nuestros resultados no son nada concluyentes, encontrando diferencias tan sólo en las dimensiones Listado de síntomas/ problemas y Efectos de la enfermedad renal del KDQOL, y teniendo en cuenta que el grupo de con CVT era minoritario ( $25 \%$ de pacientes).

La prevalencia de casos probables de ansiedad (42,6\%) y depresión $(52,6 \%)$ en nuestra muestra están en consonancia con otros estudios publicados ${ }^{26,27}$. Respecto al sexo, las mujeres presentan mayor prevalencia de ansiedad y depresión, en comparación a los hombres. General- 
mente, las mujeres tienen mayor tendencia a desarrollar trastornos ansioso-depresivos, entre otros motivos, por las fluctuaciones hormonales que sufre la mujer a lo largo de su vida, como por la presiones sociales sobre el rol de la mujer ${ }^{28}$. Por nuestro modelo cultural, las mujeres cuentan con mayor aceptación a la hora de verbalizar sus emociones; así como reconocer sus propias limitaciones. Sin embargo, al género masculino se le cohíbe más al expresar determinadas dolencias, especialmente si se trata de origen psicológico29.

En nuestro estudio, se ha podido objetivar que la ansiedad y la depresión actúan en la CVRS como elementos moduladores negativos en los pacientes en $\mathrm{HD}^{30}$, afectando a todas las dimensiones del cuestionario KDQOL y SF-36. Otros autores, no solo relacionan la ansiedad y la depresión con la CVRS, sino también con mayores tasas de mortalidad e ingresos hospitalarios en este tipo de pacientes $31,32,33$.

La edad se relacionó estadísticamente con el ICC, y ambas se relacionaron con la dimensión la Función física. Del mismo modo, cuando se separó la población geriátrica ( $>75$ años) del resto se observó que este grupo tenía un ICC mayor y que presentaban peores puntuaciones en la dimensión Función física. Esto es lógico, pues en diferentes estudios se destaca la relación estadística entre la edad y la comorbilidad, y por consiguiente peores puntuaciones en la CVRS $^{34,35}$.

El tiempo en HD afecta a la CVRS de estos pacientes. En nuestro estudio, encontramos relaciones significativas del tiempo en HD con los Efectos de la Enfermedad Renal, la Situación Laboral y el Sueño para el cuestionario KDQOL y la Salud General para el SF-36. El impacto del tiempo en TRS sobre la CVRS ha sido descrito por otros autores con unos resultados similares a los encontrados en nuestro estudio ${ }^{36,37}$, sin embargo, en otros estudios no encontraron una relación significativa ${ }^{38}$.

En cuanto a la distribución de los pacientes por centros, se puede observar que los pacientes de la UHD presentan peores puntuaciones en casi todas las variables y dimensiones de las mismas, siendo esto lógico ya que estos pacientes permanecen en esta Unidad al no tener criterios clínicos de derivación a un centro periférico.

Como principales limitaciones de este estudio señalar las propias de los estudios observacionales descriptivos, unido a la muestra, que aunque no es pequeña, no es lo suficientemente amplia como para obtener resultados más concluyentes.
A la vista de nuestros resultados, podemos concluir que los pacientes en HD tienen su CVRS disminuida, en comparación con la población general, especialmente en las dimensiones Carga de la enfermedad renal, Sueño, Salud general, Vitalidad, Función física y Rol físico. Esta disminución de la calidad de vida está relacionada con la probabilidad de padecer ansiedad y/o depresión, ya que casi la mitad de estos pacientes son susceptibles de padecer alguna de estas alteraciones o de ambas a la vez. Además de la ansiedad y/o depresión, los factores más influyentes en una peor percepción de la CVRS en estos pacientes, son el sexo femenino, la comorbilidad asociada y el tiempo en HD.

\section{Bibliografía}

1. Górriz Teruel JL, Otero González A. Impacto socio sanitario de la enfermedad renal crónica avanzada. Nefrología. 2008;28(3):7-15.

2. Sociedad Española de Nefrología. Informe de Diálisis y Trasplante 2017[Internet]. Madrid:SEN;2017 [Consultado 22.03.2019] Disponible en: https:// www.senefro.org/contents/webstructure/Informe REER_2017.pdf.

3. Gutiérrez Sánchez D, Leiva-Santos JP; Sánchez-Henández R, Gómez García R. Prevalencia y evaluación de síntomas en enfermedad renal crónica avanzada. Enferm Nefrol. 2015;18(3):228-36.

4. Frías Albañil T, Ramírez Moreno MC, Crespo Montero R. Análisis de la calidad de vida en pacientes en hemodiálisis ambulatoria y su relación con el nivel de dependencia. Enferm Nefrol. 2014;17(3):16774.

5. Seguí Gomà A, Amador Peris $P$, Alcario $R$, Belén $A$. Calidad de vida en pacientes con insuficiencia renal crónica en tratamiento con diálisis. Rev Soc Esp Enferm Nefrol. 2010;13(3):155-60. 
6. García-Llana H, Remor E, Peso G del, Selgas R. El papel de la depresión, la ansiedad, el estrés y la adhesión al tratamiento en la calidad de vida relacionada con la salud en pacientes en diálisis: revisión sistemática de la literatura. Nefrología. 2014;34(5):637-57.

7. Fernández-López JA, Fernández-Fidalgo M, Cieza Alarcos. Los conceptos de calidad de vida, salud y bienestar analizados desde la perspectiva de la Clasificación Internacional del Funcionamiento (CIF). Rev. Esp. Salud Pública. 2010;84(2):169-84.

8. Barros Higgins L, Herazo-Beltrán Y, Aroca Martínez G. Calidad de vida relacionada con la salud en pacientes con enfermedad renal crónica. Rev Fac Med. 2015;63(4):641-7.

9. Herbias Herbias L, Aguirre Soto R, Bravo Figueroa $H$, Avilés Reinoso L. Significado de calidad de vida en pacientes con terapia de hemodiálisis: un estudio fenomenológico. Enferm Nefrol. 2016;19(1):3744.

10. Gómez Villaseca L, et al. Prevalencia de ansiedad y depresión en pacientes de hemodiálisis. Enferm Nefrol. 2015;18(2):112-7.

11. Varela L, Vázquez M, Bolaños L, Alonso R. Predictores psicológicos de la calidad de vida relacionada con la salud en pacientes en tratamiento de diálisis peritoneal. Nefrología. 2011;31(1):97-106.

12. Perales Montilla C, García León A, Reyes del Paso G. Predictores psicosociales de la calidad de vida en pacientes con insuficiencia renal crónica en tratamiento de hemodiálisis. Nefrología. 2012;32(5):622-30.

13. Cruz $M$, Andrade $C$, Urrutia $M$, Draibe $S$, Nogueira Martins $L, R d$. Quality of life in patients with chronic kidney disease. CLINICS. 2011; 66(6):991-5.

14. Fan $L$, Sarnak M, Tighiouart $H$, et Al. Depression and All-Cause Mortality in Hemodialysis Patients. Am J Nephrol. 2014;40(1):12-8.

15. Villavicencio $M$, Cardona $M$, Pérez G, López $M$, Barbosa M. Ansiedad y Depresión como Indicadores de Calidad de Vida en Adultos Mayores. Revista de Psicologia da IMED. 2012;4(1):649-61.
16. Patat C, Stumm E, Kirchner R, Guido L, Barbosa D. Análisis de la calidad de vida de los usuarios de hemodiálisis. Enfermería Global. 2012;11(27):54-65.

17. Zúñiga C, Dapueto J, Müller H, Kirsten L, Alid R, Ortiz L. Evaluación de la calidad de vida en pacientes en hemodiálisis crónica mediante el cuestionario "Kidney Disease Quality of Life (KDQOL-36)". Rev Méd Chile. 2009;137(2):200-7.

18. Rodrigues Fructuoso M, Castro R, Oliveira I, Prata C, Morgado T. Quality of life in chronic kidney disease. Nefrologia. 2019;31(1):90-6.

19. Martín R. Satisfacción del paciente en hemodiálisis como criterio de calidad asistencial en enfermería. Rev Mex Urol. 2014;74(5):292-5.

20. López Toledano M, Luque Cantero I, Gómez López V, Casas Cuesta R, Crespo Montero R. La satisfacción del paciente como componente de la calidad en las unidades de diálisis. Enferm Nefrol. 2016;19(2):125-33.

21. Lew-Starowicz M, Gellert R. The Sexuality and Quality of Life of Hemodialyzed Patients-ASED Multicenter Study. J Sex Med. 2009; 6(4):1062-71.

22. Li Y, Shapiro B, Kim J, Zhang M, Porszasz J, Bross $R$ et al. Association between quality of life and anxiety, depression, physical activity and physical performance in maintenance hemodialysis patients. Chronic Dis Transl Med. 2016;2(2):110-9.

23. Capote Leyva E, Argudín Selier R, Mora González S, Capote Pereira L, Leonard Rupalé I, Moret Hernández Y. Evaluación de la calidad de vida relacionada con salud en pacientes en hemodiálisis periódica utilizando el KDQOL-SFTM. Medisur. 2015;13(4):508-16.

24. Antón-Pérez $G$, Pérez-Borges $P$, Alonso-Almán $F$, Vega-Díaz N. Accesos vasculares en hemodiálisis: un reto por conseguir. Nefrología. 2012; 32(1):1037.

25. Pelayo Alonso R, Cobo Sánchez J, Reyero López M, Sáenz de Buruaga Perea A, Tovar Rincón A, Alonso Nates $R$ et al. Repercusión del acceso vascular sobre la calidad de vida de los pacientes en tratamiento con hemodiálisis. Rev Soc Esp Enferm Nefrol.2011;14(4):242-9. 
26. Macaron G, Fahed M, Matar D, Bou-Khalil R, Kazour $F$, Nehme-Chlela $D$ et al. Anxiety, Depression and Suicidal Ideation in Lebanese Patients Undergoing Hemodialysis. Community Ment Health J. 2014;50(2):235-8.

27. Montinaro V, Iaffaldano G, Granata S, Porcelli $P$, Todarello 0, Schena $F$ et al. Emotional symptoms, quality of life and cytokine profile in hemodialysis patients. Clin Nephrol. 2010;73(1):36-43.

28. Armaly Z, Farah J, Bisharat B, Abd El Qader A, Jabbour A, Hamzi M et al. Major depressive disorders in chronic hemodialysis patients in Nazareth: identification and assessment. Neuropsychiatr Dis Treat. 2012;8:329-38.

29. Moral-de la Rubia J, Valdez-Medina J, González Arratia López-Fuentes N, González-Escobar S, Alvarado Bravo B, Gaona-Valle L. Emociones y síntomas físicos medicamente no explicados en mujeres y hombres adultos. Medicina Universitaria. 2010;12(47):96-05.

30. Theofilou P. Quality of Life in Patients Undergoing Hemodialysis or Peritoneal Dialysis Treatment. J Clin Med Res. 2011;3(3):132-8.

31. Li Y, Shapiro B, Kim J, Zhang M, Porszasz J, Bross $R$ et al. Association between quality of life and anxiety, depression, physical activity and physical performance in maintenance hemodialysis patients. Chronic Dis Transl Med. 2016;2(2):110-9.

32. Hall R, Luciano A, Pieper C, Colón-Emeric C. Association of Kidney Disease Quality of Life (KDQ0L-36) with mortality and hospitalization in older adults receiving hemodialysis. BMC Nephrology. 2018;19(1):11. 10.1186/s12882-017-0801-5.
33. Østhus TB, Preljevic VT, Sandvik L, Leivestad T, Nordhus IH, Dammen T et al. Mortality and health-related quality of life in prevalent dialysis patients: Comparison between 12-items and 36-items short-form health survey. Health and Quality of Life Outcomes. 2012;10(1):46.

34. Barbero E, Tejeda E, Herrera C, Montserrat S, Gascó $\mathrm{N}$, Junyent $\mathrm{E}$. Estudio comparativo del estado físico, mental y percepción de calidad de vida relacionada con la salud de los pacientes en diálisis. Enferm Nefrol. 2016;19(1):29-35.

35. Muñoz Sancho R, Oto Royo A, Barrio Alonso R, Fernández M. Evolución de la calidad de vida en pacientes en hemodiálisis: Estudio prospectivo a un año. Rev Soc Esp Enferm Nefrol.2006;9(1):55-8.

36. Guerra-Guerrero V, Sanhueza-Alvarado 0, Cáceres-Espina $M$. Calidad de vida de personas en hemodiálisis crónica: relación con variables sociodemográficas, médico-clínicas y de laboratorio. Rev Latino-Am Enfermagem. 2012;20(5):838-46.

37. Ruiz de Alegría Fernández de Retana $B$, Basabe Barañano N, Fernández Prado E, Baños Baños C, Nogales Rodríguez M, Echebarri Escribano M et al. Cambios en las estrategias de afrontamiento en los pacientes de diálisis a lo largo del tiempo. Rev Soc Esp Enferm Nefrol. 2009;12(1):11-7.

38. Urzúa A, Pavlov R, Cortés R, Pino V. Factores Psicosociales Relacionados con la Calidad de Vida en Salud en Pacientes Hemodializados. Terapia psicológica. 2011;29(1):135-40.

Este artículo se distribuye bajo una Licencia Creative Commons Atribución-NoComercial 4.0 Internacional. https://creativecommons.org/licenses/by-nc/4.0/

Open Access (c) (7) (3) 